\title{
Correction to: Hard-material Adhesion: Which Scales of Roughness Matter?
}

\author{
L. A. Thimons ${ }^{1} \cdot$ A. Gujrati ${ }^{1}$ A. Sanner ${ }^{2,3} \cdot$ L. Pastewka $^{2,3} \cdot$ T. D. B. Jacobs ${ }^{1}$ \\ Published online: 13 September 2021 \\ (c) Society for Experimental Mechanics 2021 \\ Correction to: Experimental Mechanics \\ https://doi.org/10.1007/s11340-021-00733-6
}

This article was updated to replace an uncorrected version that was inadvertently posted.

Publisher's Note Springer Nature remains neutral with regard to jurisdictional claims in published maps and institutional affiliations.

The original article can be found online at https://doi.org/10.1007/ s11340-021-00733-6.

T. D. B. Jacobs

tjacobs@pitt.edu

1 Department of Mechanical Engineering and Materials Science, University of Pittsburgh, Pittsburgh, PA 15261, USA

2 Department of Microsystems Engineering, University of Freiburg, Georges-Köhler-Allee 103, 79110 Freiburg, Germany

3 Cluster of Excellence livMatS, Freiburg Center for Interactive Materials and Bioinspired Technologies, University of Freiburg, Georges-Köhler-Allee 105, 79110 Freiburg, Germany 